opment of macrophage and granulocyte colonies from single, normal undifferentiated cells requires constant interaction, presumably at the cell surface, with at least one specific mediator. The mediator is a protein with a molecular weight of about 65,000 that requires a small co-factor for activity (replaceable in certain conditions by adenine deriva. tives including cyclic AMP), and is released by various types of cells in culture into the tissue culture medium. The myeloid leukaemias contain two types of cells, only one of which can be induced to undergo normal differentiation. Again MG1 is needed but the leukaemic cells supply their own, as yet unidentified, co-factor. A clear difference between the two cell types was found by the ability only of the differentiation-competent cells to 'cap' con A molecules bound to their surface. In agreement with older results with normal lymphocytes, normal and virally transformed fibroblasts, clustering or 'cap' formation induced by cross-linking of membrane sites by con A was blocked by metabolic inhibitors, 'freezing' the membrane with aldehyde fixatives or simply by dropping the temperature. Again as often found in such cases, trypsinisation of differentiationincompetent cells increased the mobility of con A binding sites and caps were formed.

Although thousands of similar observations have been described in a wide variety of systems, the molecular basis for increased mobility of surface sites is still obscure. A direct effect on the viscosity of the membrane lipids seems unlikely. The observation of Inbar et al. that 'cap' formation was helped by adding antibodies against con $\mathrm{A}$, or of glycogen, after con A binding suggests that the surface sites were simply too widely spaced to form unaided the necessary cross-linked matrix with the lectin. Edelman et al. (Proc. natn. Acad. Sci., U.S.A., 70, 1442; 1973) suggest that the movement of surface sites is controlled by proteins attached to the inner cytoplasmic face of the surface membrane, the activity or concentration of which is altered. Certainly interaction between these internal proteins and external surface glycoproteins, that may span the cytoplasmic membrane, seems probable.

Whatever the true explanation turns out to be, however, the conclusion of Inbar et al. is that a difference in membrane fluidity of specific sites may explain differences in the ability of leukaemic cells to respond to the differentiation-inducing stimulus of MG1. It is now of some interest to correlate more directly the mobility of cellular MG1 receptor sites with the differentiation competence of those cells. Similarly one is curious about the behaviour of normally differentiation-incompetent cells in an environment containing MG1 and low amounts of trypsin. Whatever the outcome there is no doubt that the experimental substance of this report will appear again and again in discussions of mechanisms controlling other facets of cell development.

\section{INSECT METAMORPHOSIS Competence to Mouli}

from our Insect Physiology Correspondent IT has long been known that when the 'imaginal disks' from young larvae of Drosophila are transplanted into larval hosts that are ready to pupate, they are unable to differentiate into adult structures. They gradually become competent for imaginal differentiation towards the end of the second instar, 50 to $70 \mathrm{~h}$ after hatching. The stimulus for the initiation of metamorphosis is a high concentration of the moulting hormone ecdysone.

In a short but interesting paper Mindek and Nöthiger (J. Insect Physiol., 19. 1711 ; 1973) describe a renewed attempt to discover the nature of the changes which result in the acquisition of competence. The experiments consisted in exposing eye-antennal disks removed from donor larvae $53 \mathrm{~h}$ old, which are completely incompetent for metamorphosis at this stage, and treating them in various ways before testing their competence by implantation into mature larvae, just before pupation at $120 \mathrm{~h}$. Disks were maintained in adults receiving normal food, in adults 'starved' by feeding on sugar alone, or adults starved for a time and then given normal food. The results show that competence to respond to ecdysone does not simply require a minimum number of cells (a 'critical mass'); nor is it determined by the time elapsed since the formation of the disk.

The authors conclude that cell divisions are the prerequisite for gaining new developmental possibilities and that a minimum number of cell divisions is needed to allow the necessary changes in the developmental programming of the cells.

\section{PROTEIN SYNTHESIS}

\section{Control Mechanisms}

from a Correspondent

EXPERIMENTS with mammalian cells in culture have revealed many diverse situations in which the rate of initiation of protein synthesis is greatly reduced. Besides the now classical example of rabbit reticulocytes deprived of haem, inhibition of initiation is seen when cells enter mitosis, are shifted to $42^{\circ} \mathrm{C}$, starved of serum or an essential amino acid, infected with poliovirus or exposed to anaerobiosis. In some cases the inhibition is selective; more often the inhibition appears to affect all proteins to the same extent, but, apart from the inhibition of globin synthesis in the absence of haem, very little is known about the underlying control mechanisms. One reason for this is that extracts of cells other than the reticulocyte show almost no initiation on endogenous messenger RNA, so that normal and inhibited cells cannot easily be compared in vitro. This impasse has been present for a long time and only

\title{
Secondary Structure in an RNA Molecule
}

As soon as the primary structure of an RNA molecule is elucidated its discoverers set about the happy task of looking for possible secondary structures. When these are published they have tended to elicit alternative secondary structures from competing workers. How can a choice between these alternatives be made? The answer requires a means of assessing the free energy of a given structure and this in turn requires basic information concerning the free energy changes attendant on the formation of the various features-such as base pairs, hairpin loops, interior loops, bulgeswhich constitute the structure. Each new investigation of the thermodynamics of the secondary structure in oligonucleotides and other simple models leads to a refinement in these calculations.

In their latest paper Tinoco et al. (see Nature New Biology next Wednesday, November 14) amass recent results for the sequence dependence of the free energy of formation of base pairs as well as that of various types of bulge and loop. The result is a table of free energy changes from which the total change on forming a given structure from the random coil state at $25^{\circ} \mathrm{C}$ can be calculated. Each entry in the table, it is claimed, is good to $10 \%$ but the uncertainty in the final figure may be somewhat greater; the authors also point out that the algorithm they describe does not take any account of tertiary structure. Nevertheless, it may serve to eliminate some of the more improbable models even if it does not allow a definitive assessment as to which is the most stable.

Tinoco et al. suggest that their work may lead to attempts to refine the theory and the experimental data on which it is based and even lead to investigation of temperature dependences which would be highly relevant to the interpretation of melting curves. 